\title{
Effects of robot-assisted gait training on lower extremity strength, functional independence, and walking function in men with incomplete traumatic spinal cord injury
}

\author{
Melike Mıdık ${ }^{1} \oplus$, Nurdan Paker ${ }^{1} \oplus$, Derya Buğdayc1 ${ }^{1} \oplus$, Ali Can $\operatorname{Mıd}^{2} k^{2} \oplus$ \\ ${ }^{1}$ Department of Physical Medicine and Rehabilitation, Istanbul Physical Therapy Rehabilitation Training and Research Hospital, Istanbul, Turkey \\ ${ }^{2}$ Department of Emergency Medicine, Çanakkale State Hospital, Çanakkale, Turkey
}

Received: July 06, 2018 Accepted: November 28, 2018 Published online: March 03, 2020

\begin{abstract}
Objectives: The aim of this study was to investigate the effects of robotic treadmill training on lower extremity motor function, functional independence, and walking in men with traumatic incomplete spinal cord injury (SCI).

Patients and methods: A total of 30 consecutive male patients (mean age 36.6 years; range, 19 to 53 years) with traumatic incomplete SCI who were admitted to our inpatient clinic of the rehabilitation hospital were included in this study. All patients had conventional rehabilitation for five days weekly. The patients were randomized into two groups as the robot-assisted gait training (RAGT) group ( $\mathrm{n}=15$ ) for $30 \mathrm{~min}$ for three times a week for a total of five weeks and the conventional rehabilitation group (control group, n=15). The patients were evaluated sociodemographically and neurologically at baseline. Lower Extremity Motor Score (LEMS), Spinal Cord Independence Measurement-III (SCIM-III), and Walking Index for Spinal Cord Injury-II (WISCI-II) were used at baseline, at the end of the fifth week, and at three months after the treatment.

Results: The mean disease duration was $23 \pm 33$ months in the RAGT group and $28.6 \pm 17$ months in the conventional rehabilitation group $(\mathrm{p}>0.05)$. A significant improvement was observed in the LEMS, WISCI-II, and SCIM-III scores after the treatment in both groups ( $\mathrm{p}<0.05)$. The improvement in the LEMS scores was significantly higher in the RAGT group at the end of the fifth week and at three months ( $\mathrm{p}=0.017)$. The SCIM-III scores were also significantly improved in the RAGT group at the end of the fifth week and at three months (p=0.038).

Conclusion: Our study results show that conventional rehabilitation is useful in terms of the improvement in the lower extremity motor function, walking, and functional status in men with incomplete SCI. Additional RAGT provides a better improvement in the lower extremity motor function and functional independence.
\end{abstract}

Keywords: Functional status, robot-assisted gait training, Spinal Cord Independence Measurement-III, spinal cord injury, walking.

Most of the spinal cord injury (SCI) patients experience motor function loss which result in reduced mobility. ${ }^{[1]}$ Restoring walking function is of high priority to patients with SCI. ${ }^{[2]}$ In gait rehabilitation, different modalities such as manually-assisted treadmill training and manually-assisted overground training, and body weight-supported gait training can be used. ${ }^{[3]}$ The rehabilitation protocol of SCI usually consists of a combination of exercises and different technologyaided modalities. Robot-assisted gait training (RAGT) is one of the modern rehabilitation tools that may help to erect locomotion. ${ }^{[4]}$ It can be used both in acute and chronic SCI patients and provides improvement in walking distance, leg strength, functional mobility, and functional independence levels in acute SCI patients. ${ }^{[5]}$

For SCI patients with an injury duration of less than a year, both RAGT and body weight-supported treadmill training (BWSTT) provide a better improvement in gait parameters than conventional methods. ${ }^{[6]}$ In chronic SCI, RAGT may help to increase in walking distance with improved functional mobility. ${ }^{[5,7]}$ On the other hand, it has been shown that BWSTT with functional

Corresponding author: Melike Mıdık, MD. İstanbul Fizik Tedavi Rehabilitasyon Eğitim ve Araştırma Hastanesi, Fiziksel Tıp ve Rehabilitasyon Kliniği, 34186 Bahçelievler, İstanbul, Türkiye. e-mail: dr_melike84@hotmail.com 
electrical stimulation (FES) and overground training with FES/body weight support offer a higher increase in ambulation than that of BWSTT with manual assistance, robotic gait training, or conventional physiotherapy methods during chronic stage. ${ }^{[6]}$ Additional RAGT to the conventional rehabilitation usually improves walking function in patients with SCI. ${ }^{[8]}$

In the present study, we aimed to compare the results of additional RAGT to conventional rehabilitation versus conventional rehabilitation alone on lower extremity motor functions, functional independence, and walking function in men with traumatic incomplete SCI.

\section{PATIENTS AND METHODS}

This prospective, randomized-controlled study was conducted at Istanbul Physical Therapy Rehabilitation Training and Research Hospital between September 2011 and February 2013. A total of 30 consecutive male patients (mean age 36.6 years; range, 19 to 53 years) with traumatic incomplete SCI who were admitted to our inpatient rehabilitation clinic were included in this study. Inclusion criteria were as follows: age $\geq 18$ years, having traumatic incomplete SCI, being male, an injury duration of at least 12 weeks, and a Lower Extremity Motor Score (LEMS) of $\geq 10$. Patients with SCI and cardiovascular disease, contractures or deformities in the lower extremities, or pressure ulcers were excluded. Sociodemographic characteristics were recorded, and neurological examination was performed at baseline. The American Spinal Injury Association (ASIA) Impairment Scale was used to obtain motor and sensory scores. A written informed consent was obtained from each patient. The study protocol was approved by the Bakırköy Dr. Sadi Konuk Training and Research Hospital Ethics Committee. The study was conducted in accordance with the principles of the Declaration of Helsinki.

All patients were randomized into two groups using block randomization. The physician who was responsible for the patient evaluation was unblinded; however, the physiotherapist who was responsible for the rehabilitation protocol was blinded. All patients received regular physiotherapy for five times a week for a total of five weeks. The first group (RAGT group, $\mathrm{n}=15$ ) received additional RAGT and the conventional rehabilitation group $(\mathrm{n}=15)$ received conventional rehabilitation protocol. We used the Lokomat Prodevice (Hocoma Inc., Zurich, Switzerland) for RAGT. It includes a supported body weight system with robotic gait orthosis used in combination with a treadmill. It was administered for three times a week for a total of five weeks under the supervision of an educated physiotherapist and each session lasted $30 \mathrm{~min}$. Baseline treadmill speed was set to $1.5 \mathrm{~km} / \mathrm{h}$ and was increased as much as the patient could tolerate. At the beginning, half (50\%) of the patient's weight was supported by a body weight support system, but it was decreased then, as much as the patient could tolerate.

The control group received only conventional rehabilitation which consisted of range of motion exercises, strengthening exercises, body stabilization, self-care ability, and ground walking training. All patients were evaluated using the LEMS, Spinal Cord Independence Measurement-III (SCIM-III), and Walking Index for Spinal Cord Injury-II (WISCI-II) at baseline $\left(t_{1}\right)$, at the end of the treatment at five weeks $\left(t_{2}\right)$, and at three months after the treatment $\left(\mathrm{t}_{3}\right)$. The patients were discharged after the treatment. The patients did not receive any treatment from Week 5 to Month 3 .

\section{Outcome measures}

The LEMS is derived from the strength of key muscles in lower extremity, which was originally described by the ASIA. These muscles include knee extensors, hip flexors, ankle dorsiflexors, ankle plantar flexors, and long toe extensors of both lower extremities. The scores range from 0 to 50 for both lower extremities. Lower scores indicate weak motion, while higher scores indicate better strength.

The SCIM-III was used to evaluate functional independence in both groups before rehabilitation $\left(t_{1}\right)$, immediately after $\left(t_{2}\right)$, and three months after rehabilitation $\left(t_{3}\right)$. The SCIM-III is a reliable tool which measures the independence level after SCI. ${ }^{[9]}$ Total SCIM-III score ranges from 0 to 100 , which includes subitems such as personal care $(0-20)$, respiration and sphincter management (0-40), and mobility (0-40). Lower scores indicate decreased independence level. The Turkish adaptation of the SCIM-III has been conducted. ${ }^{[10]}$

Both WISCI and WISCI-II are used to measure walking function. ${ }^{[11,12]}$ However, in the present study, we used the WISCI-II to evaluate the impairment of walking function. The scores range from 0 to 20 . Zero indicates the most severe impairment, while 20 indicate the least severe impairment.

\section{Statistical analysis}

Power analysis was performed using the $G^{\star}$ Power version 3.1.7 software (Heinrich Heine University 


\begin{tabular}{|c|c|c|c|c|c|c|c|c|c|c|c|}
\hline & & & Demograp & $\mathrm{c}$ and $\mathrm{cl}$ & $\begin{array}{l}\text { TABLE } 1 \\
\text { hical charac }\end{array}$ & teris & cs of & atients & & & \\
\hline & & & RAC & & & & & Cont & & & \\
\hline & $\mathrm{n}$ & $\%$ & Mean \pm SD & Median & $\begin{array}{l}1^{\text {st }} \text { and } 3^{\text {rd }} \\
\text { quartile }\end{array}$ & $\mathrm{n}$ & $\%$ & Mean \pm SD & Median & $\begin{array}{l}1^{\text {st }} \text { and } 3^{\text {rd }} \\
\text { quartile }\end{array}$ & $p$ \\
\hline Age (year) & & & $35.4 \pm 12.1$ & & & & & $37.9 \pm 10.0$ & & & $0.547^{\star}$ \\
\hline $\mathrm{BMI}\left(\mathrm{kg} / \mathrm{m}^{2}\right)$ & & & $24.6 \pm 3.9$ & & & & & $26.5 \pm 3.1$ & & & $0.153^{*}$ \\
\hline Time after SCI (month) & & & & 5 & 4,30 & & & & 24 & 17,44 & $0.029^{* *} \S$ \\
\hline Etiology & & & & & & & & & & & $0.719 \dagger$ \\
\hline Traffic accident & 4 & 26.7 & & & & 4 & 26.7 & & & & \\
\hline Falls & 9 & 60 & & & & 7 & 46.7 & & & & \\
\hline Others & 2 & 13.3 & & & & 4 & 26.7 & & & & \\
\hline Injury severity & & & & & & & & & & & $0.143 \ddagger$ \\
\hline AIS C & 6 & 40 & & & & 10 & 66.7 & & & & \\
\hline AIS D & 9 & 60 & & & & 5 & 33.3 & & & & \\
\hline Injury level & & & & & & & & & & & $\mathrm{e} 0.080 \mathrm{~g}$ \\
\hline $\mathrm{T} 12$ & 6 & 40 & & & & 1 & 6.7 & & & & \\
\hline L1-3 & 9 & 60 & & & & 14 & 93.3 & & & & \\
\hline
\end{tabular}

Düsseldorf, Düsseldorf, Germany) and the sample size was calculated. To determine the effect size at alpha $=0.05$ (type 1 error) with $80 \%$ power (1-beta), minimum sample size to be chosen was 14 participants in each group. However, the study was completed with 15 participants for the patient group and 15 participants for the control group.

Statistical analysis was performed using the IBM SPSS for Mac version 20.0 software (IBM Corp., Armonk, NY, USA). The Shapiro-Wilk test was used to determine whether the data were distributed normally. Independent samples t-test and Mann-Whitney U test for normal and non-normal distribution estimated the significance of differences between groups, respectively. Fisher-Freeman-Halton exact test, Fisher's exact test and Pearson's chi-square test were used to test the significance of differences between categorical variables. The results for parametric tests were expressed in mean \pm standard deviation (SD), while the results for non-parametric test were expressed in median ( $1^{\text {st }}$ and $3^{\text {rd }}$ quartile). Three separate generalized linear mixed models (GLMM) were used to analyze the effects of inter-subject (RAGT vs. control) and of intrasubject $\left(t_{1}, t_{2}\right.$, and $\left.t_{3}\right)$ factors on the LEMS, WISCI-II, and SCIM variables. The Bonferroni corrected post-hoc tests were used, where appropriate. A $p$ value of $<0.05$ was considered statistically significant.

\section{RESULTS}

Clinical characteristics of the patients and controls are shown in Table 1. Median disease duration for the RAGT and control group was five and 24 months, respectively. There was a statistically significant improvement in the LEMS, WISCI-II, and SCIM-III

\begin{tabular}{|llcc|}
\hline \multicolumn{4}{c}{ TABLE 2 } \\
Generalized linear mixed model results for LEMS, \\
WISCI-II, and SCIM-III
\end{tabular}




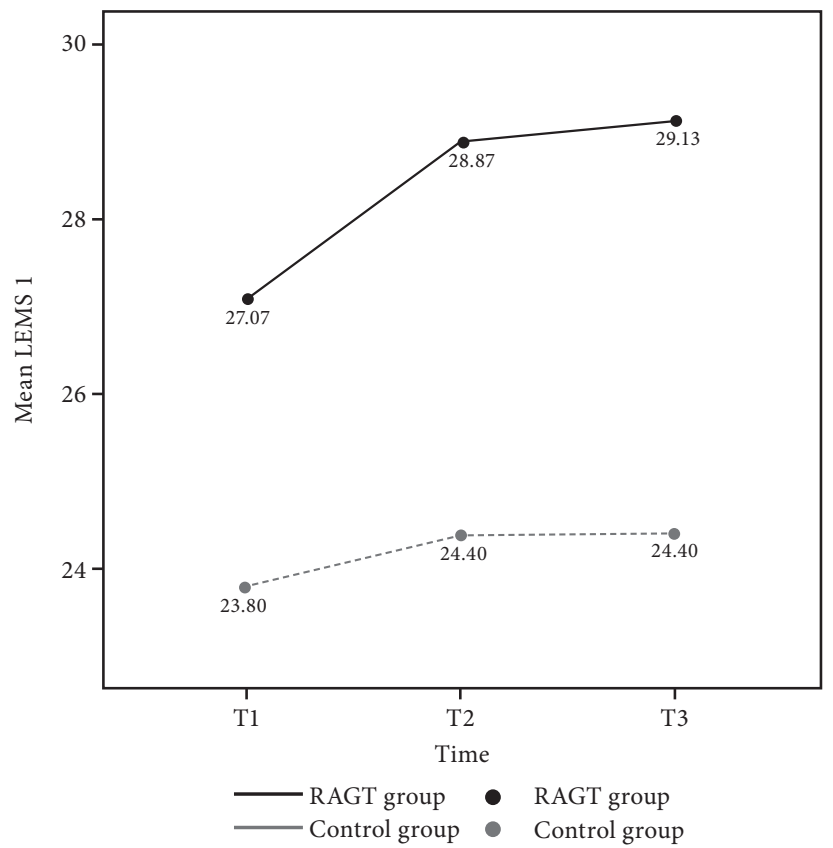

Figure 1. Change in Lower Extremity Motor Scores between groups.

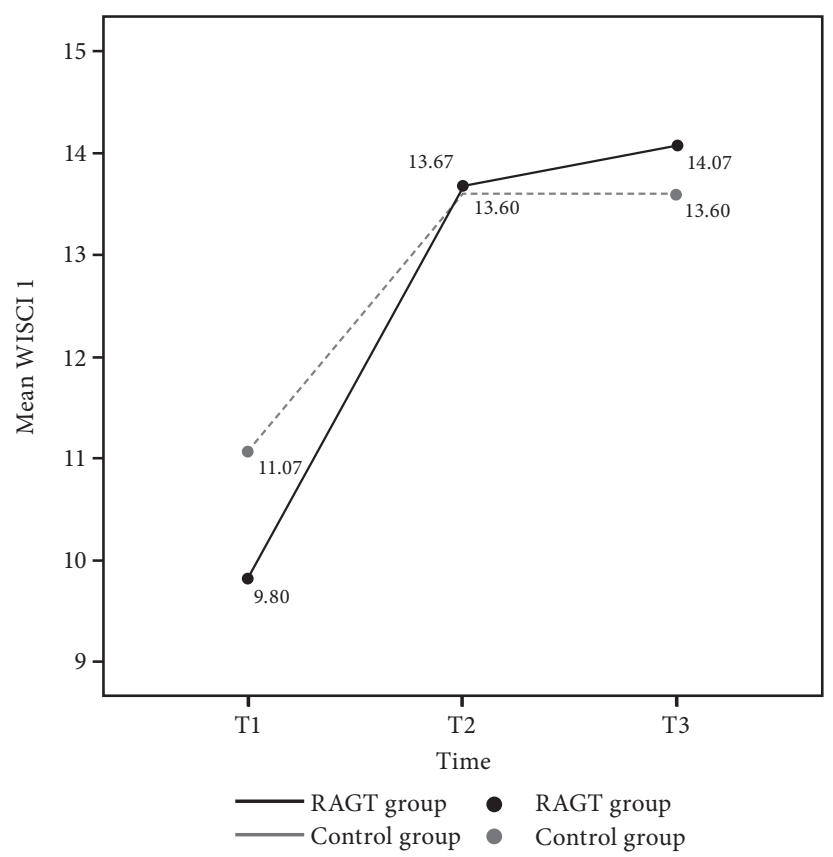

Figure 2. Changes in Walking Index for Spinal Cord Injury-II scores between groups.

scores after the treatment in both groups (Table 2). However, higher LEMS were found at $t_{2}(p=0.003)$ and $t_{3}(p=0.003)$, compared to baseline values in the RAGT group. The control group had also higher LEMS at $\mathrm{t}_{2}(\mathrm{p}=0.014)$ and $\mathrm{t}_{3}(\mathrm{p}=0.014)$, compared to baseline.

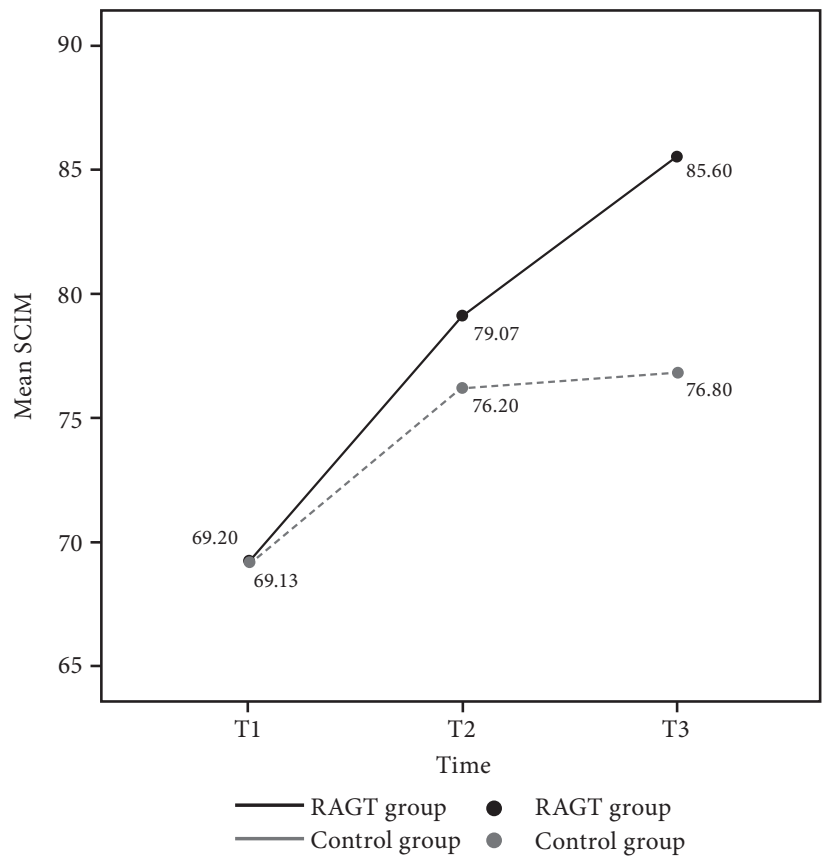

Figure 3. Change in Spinal Cord Independence Measurement-III scores between groups.

\begin{tabular}{|c|c|c|c|c|}
\hline \multicolumn{5}{|c|}{$\begin{array}{c}\text { TABLE } 3 \\
\begin{array}{c}\text { Evaluation for LEMS, WISCI-II, and SCIM-III according } \\
\text { to timepoints between groups }\end{array}\end{array}$} \\
\hline & \multirow[b]{2}{*}{ Time } & \multirow{2}{*}{$\begin{array}{c}\text { RAGT } \\
\text { Mean } \pm \text { SE }\end{array}$} & \multirow{2}{*}{$\begin{array}{c}\text { Control } \\
\text { Mean } \pm \text { SE }\end{array}$} & \multirow[b]{2}{*}{$\begin{array}{l}\text { Adjusted } \\
p \text { values }\end{array}$} \\
\hline & & & & \\
\hline \multirow[t]{6}{*}{ LEMS } & $\mathrm{T}_{1}$ & $27.1 \pm 3.3$ & $23.8 \pm 2.3$ & 0.419 \\
\hline & $\mathrm{T}_{2}$ & $28.9 \pm 3.6$ & $24.4 \pm 2.2$ & 0.289 \\
\hline & $\mathrm{T}_{3}$ & $29.1 \pm 3.7$ & $24.4 \pm 2.2$ & 0.270 \\
\hline & $\mathrm{T}_{2}-\mathrm{T}_{1}$ & $1.8 \pm 0.4^{\star *}$ & $0.6 \pm 0.2^{* *}$ & 0.061 \\
\hline & $\mathrm{T}_{3}-\mathrm{T}_{1}$ & $2.1 \pm 0.5^{\star *}$ & $0.6 \pm 0.2^{* *}$ & $0.049^{*}$ \\
\hline & $\mathrm{T}_{3}-\mathrm{T}_{2}$ & $0.3 \pm 0.2$ & $0 \pm 0$ & 0.459 \\
\hline \multirow[t]{6}{*}{ WISCI } & $\mathrm{T}_{1}$ & $9.8 \pm 1.4$ & $11.0 \pm 1.1$ & 0.464 \\
\hline & $\mathrm{T}_{2}$ & $13.7 \pm 1.1$ & $13.6 \pm 1.0$ & 0.963 \\
\hline & $\mathrm{T}_{3}$ & $14.1 \pm 1.1$ & $13.6 \pm 1.0$ & 0.746 \\
\hline & $\mathrm{T}_{2}-\mathrm{T}_{1}$ & $3.9 \pm 0.8^{\star *}$ & $2.5 \pm 0.5^{\star *}$ & 0.178 \\
\hline & $\mathrm{T}_{3}-\mathrm{T}_{1}$ & $4.3 \pm 1.0^{\star *}$ & $2.5 \pm 0.5^{\star *}$ & 0.139 \\
\hline & $\mathrm{T}_{3}-\mathrm{T}_{2}$ & $0.4 \pm 0.3$ & $0 \pm 0$ & 0.153 \\
\hline \multirow[t]{6}{*}{ SCIM } & $\mathrm{T}_{1}$ & $69.1 \pm 4.9$ & $69.2 \pm 3.0$ & 0.991 \\
\hline & $\mathrm{T}_{2}$ & $79.1 \pm 4.6$ & $76.2 \pm 2.4$ & 0.579 \\
\hline & $\mathrm{T}_{3}$ & $85.6 \pm 2.2$ & $76.8 \pm 2.4$ & $0.008^{\star *}$ \\
\hline & $\mathrm{T}_{2}-\mathrm{T}_{1}$ & $9.9 \pm 2.5^{\star *}$ & $7.0 \pm 1.3^{\star *}$ & 0.326 \\
\hline & $\mathrm{T}_{3}-\mathrm{T}_{1}$ & $16.5 \pm 3.2^{\star *}$ & $7.6 \pm 1.5^{\star *}$ & 0.127 \\
\hline & $\mathrm{T}_{3}-\mathrm{T}_{2}$ & $6.5 \pm 5.1$ & $0.6 \pm 0.3$ & 0.271 \\
\hline
\end{tabular}


There was no significant difference in terms of baseline LEMS scores between two groups (Figure 1).

In addition, we found higher SCIM-III scores in the RAGT group, compared to the control group at $t_{2}(p=0.03)$ and $t_{3}(p=0.01)$. Both groups had higher WISCI-II scores at $\mathrm{t}_{2}(\mathrm{p}=0.002$ and $\mathrm{p}=0.003$, respectively) and $t_{3}(p=0.002$ and $p=0.003$, respectively) (Figures 2 and 3).

The LEMS and SCIM-III scores were statistically significantly higher in the RAGT group than the control group at $t_{2}$ and $t_{3}$. The patients in the RAGT group had statistically significantly higher LEMS than the control group at $t_{2}(p=0.02)$ and $t_{3}(p=0.017)$. However, there was no statistically significant difference in the SCIM-III scores at $t_{2}$ and $t_{3}$. Also, there was no significant difference in the improvement in the WISCI-II scores between the groups at $t_{2}$ and $t_{3}$ (Table 3).

\section{DISCUSSION}

In the present study, we evaluated the outcomes of RAGT and conventional rehabilitation on lower extremity motor functions, functional independence, and walking function in men with traumatic incomplete SCI. The patients in both groups showed a significant improvement in terms of LEMS, functional independence, and ambulation; however, the increase in the LEMS and functional independence scores was significantly higher in the RAGT group.

In their study, Wirz et al. ${ }^{[1]}$ reported that chronic incomplete SCI patients receiving RAGT had improved LEMS scores at four and eight weeks after the treatment. On the contrary, in another study, walking speed remained unchanged after RAGT in patients with chronic incomplete SCI. ${ }^{[13]}$ This discrepancy between the studies may result from the different injury durations. Also, both the patient and RAGT-related characteristics affect the treatment results. Horny et al. ${ }^{[14]}$ reported a significant improvement in the LEMS in patients receiving RAGT; however, they were unable to find any significant differences between the RAGT and BWSTT groups. Therefore, the results of our study are consistent with the literature, indicating significantly improved LEMS scores after the treatment. The RAGT was also shown to be useful for restoring walking function in SCI patients. ${ }^{[15]}$

In our study, the improvement in the functional independence was greater in the RAGT group compared to the control group. The patients in the RAGT group had significantly better SCIM-III scores immediately after the treatment and at three months than the control group. Similarly, Schwartz et al. ${ }^{[16]}$ reported significantly higher SCIM-III scores in patients with SCI after RAGT.

One of the important findings of the present study is significantly higher WISCI-II scores after the treatment in both groups. However, there was no significant difference in terms of the WISCI-II scores between two groups. Both RAGT and conventional physiotherapy with ground walking training were effective for the improvement of walking function. Schwartz et al. ${ }^{[16]}$ also reported an non-significant improvement in the WISCI-II scores in the RAGT patients. Wirz et al. ${ }^{[7]}$ concluded that chronic incomplete SCI patients had an improvement in the WISCI-II scores and walking speed after RAGT. Shin et al. ${ }^{[17]}$ reported that improvement in the LEMS, SCIM-III, and WISCI-II scores were found in the RAGT and conventional physiotherapy groups; however, RAGT with conventional rehabilitation provided better improvement in terms of ambulation in patients with subacute incomplete SCI. Moreover, they reported no significant difference in the functional independence level and LEMS scores between the conventional and robotic treatment groups in which RAGT was consisted of 40-min sessions for three times a week for a total of four weeks.

Furthermore, Hwang et al. ${ }^{[18]}$ concluded that 30-min RAGT sessions for five times a week for a total of 20 sessions was useful in terms of ambulation. The improvement was higher among younger patients and those with shorter injury duration who had subacute incomplete motor SCI. In our study, similarly, we found a significant improvement in terms of ambulation in both conventional rehabilitation and RAGT groups after 30-min sessions for three times a week for a total of five weeks.

Nonetheless, this study has some strengths and limitations. Prospective design and regular follow-up are the main strengths of the study. However, shorter injury duration in the RAGT group than the conventional rehabilitation group is the main limitation arising from the randomization. The second limitation is longer time in a day which was spent for rehabilitation in the RAGT group due to additional RAGT. Therefore, shorter injury duration and longer daily treatment period may lead to a better improvement in terms of lower extremity motor functions and functional independence in the RAGT group.

In conclusion, RAGT with conventional rehabilitation provides a greater improvement in terms 
of the lower extremity motor function and functional independence in male patients with incomplete SCI. However, further large-scale, prospective studies with optimal session duration, session number, and timing of RAGT are needed to confirm these findings.

\section{Declaration of conflicting interests}

The authors declared no conflicts of interest with respect to the authorship and/or publication of this article.

\section{Funding}

The authors received no financial support for the research and/or authorship of this article.

\section{REFERENCES}

1. Bishop L, Stein J, Wong CK. Robot-aided gait training in an individual with chronic spinal cord injury: a case study. J Neurol Phys Ther 2012;36:138-43.

2. Ditunno PL, Patrick M, Stineman M, Ditunno JF. Who wants to walk? Preferences for recovery after SCI: a longitudinal and cross-sectional study. Spinal Cord 2008;46:500-6.

3. Swinnen E, Duerinck S, Baeyens JP, Meeusen R, Kerckhofs E. Effectiveness of robot-assisted gait training in persons with spinal cord injury: a systematic review. J Rehabil Med 2010;42:520-6.

4. Hayes SC, James Wilcox CR, Forbes White HS, Vanicek $\mathrm{N}$. The effects of robot assisted gait training on temporalspatial characteristics of people with spinal cord injuries: A systematic review. J Spinal Cord Med 2018;41:529-43.

5. Nam KY, Kim HJ, Kwon BS, Park JW, Lee HJ, Yoo A. Robotassisted gait training (Lokomat) improves walking function and activity in people with spinal cord injury: a systematic review. J Neuroeng Rehabil 2017;14:24.

6. Morawietz C, Moffat F. Effects of locomotor training after incomplete spinal cord injury: a systematic review. Arch Phys Med Rehabil 2013;94:2297-308.

7. Wirz M, Zemon DH, Rupp R, Scheel A, Colombo G, Dietz $\mathrm{V}$, et al. Effectiveness of automated locomotor training in patients with chronic incomplete spinal cord injury: a multicenter trial. Arch Phys Med Rehabil 2005;86:672-80.
8. Özbudak Demir S. Robot-Assisted Gait Training for Patients with Spinal Cord Injury. Turk J Phys Med 2015;61:S37-S44.

9. Catz A, Itzkovich M, Tesio L, Biering-Sorensen F, Weeks C, Laramee MT, et al. A multicenter international study on the Spinal Cord Independence Measure, version III: Rasch psychometric validation. Spinal Cord 2007;45:275-91.

10. Kesiktas N, Paker N, Bugdayci D, Sencan S, Karan A, Muslumanoglu L. Turkish adaptation of Spinal Cord Independence Measure--version III. Int J Rehabil Res 2012;35:88-91.

11. Ditunno JF Jr, Ditunno PL, Graziani V, Scivoletto G, Bernardi M, Castellano V, et al. Walking index for spinal cord injury (WISCI): an international multicenter validity and reliability study. Spinal Cord 2000;38:234-43.

12. Ditunno JF Jr, Ditunno PL, Scivoletto G, Patrick M, Dijkers $\mathrm{M}$, Barbeau $\mathrm{H}$, et al. The Walking Index for Spinal Cord Injury (WISCI/WISCI II): nature, metric properties, use and misuse. Spinal Cord 2013;51:346-55.

13. Field-Fote EC, Roach KE. Influence of a locomotor training approach on walking speed and distance in people with chronic spinal cord injury: a randomized clinical trial. Phys Ther 2011;91:48-60.

14. Hornby TG, Zemon DH, Campbell D. Robotic-assisted, body-weight-supported treadmill training in individuals following motor incomplete spinal cord injury. Phys Ther 2005;85:52-66.

15. Tefertiller C, Pharo B, Evans N, Winchester P. Efficacy of rehabilitation robotics for walking training in neurological disorders: a review. J Rehabil Res Dev 2011;48:387-416.

16. Schwartz I, Sajina A, Neeb M, Fisher I, Katz-Luerer M, Meiner Z. Locomotor training using a robotic device in patients with subacute spinal cord injury. Spinal Cord 2011;49:1062-7.

17. Shin JC, Kim JY, Park HK, Kim NY. Effect of roboticassisted gait training in patients with incomplete spinal cord injury. Ann Rehabil Med 2014;38:719-25.

18. Hwang S, Kim HR, Han ZA, Lee BS, Kim S, Shin H, et al. Improved gait speed after robot-assisted gait training in patients with motor incomplete spinal cord injury: A preliminary study. Ann Rehabil Med 2017;41:34-41. 